\title{
Mitochondrial DNA Point Mutation at Nucleotide Pair 3316 in a Japanese Family with Heterogeneous Phenotypes of Diabetes
}

\author{
Shigeru NAKANO, Masataka FUKUDA, Fumitake HOTTA, Tomohiko ITO, \\ TAKashi ISHII, MitsutaKa KITAZAWA, MaKoto NISHIZAWA, ToshiKaZU KIGOSHI, \\ HiROAKI KAKINUMA*, HIROAKI TAKAHASHI*, AND KENZO UCHIDA \\ Division of Endocrinology, Department of Internal Medicine, and ${ }^{*}$ Department of Pediatrics, \\ Kanazawa Medical University, Ishikawa 920-0293, Japan
}

\begin{abstract}
A mitochondrial DNA (mtDNA) point mutation at nucleotide pair ( $\mathrm{np}$ ) 3316 has been reported in relation to diabetes. We recently encountered a non-obese family with this type of mutation. The proband in the affected family, a 49-year-old woman who had been previously diagnosed as having an insulin-requiring non-insulin-dependent diabetes mellitus (NIDDM), was referred to our hospital for treatment of diabetic gangrene in her left foot. Her insulin secretory capacity was markedly reduced, but the insulin sensitivity evaluated by the euglycemic hyperinsulinemic clamp technique was normal. In addition, her serum lactate level was markedly increased after a 5 min ambulation, although her serum pyruvate and ketones remained within the normal range. Twenty-year-old twin sons had been treated with insulin since the age of 7 , when both were diagnosed with insulin-dependent diabetes mellitus (IDDM). The proband's mother, a 68-year-old, was nondiabetic at this time. MtDNA analysis revealed a point mutation at $\mathrm{np} 3316$ in all family members, which was homoplasmic for the mutation on a photograph of agarose gel electrophoresis containing ethidium bromide under ultraviolet light. This mutation seemed to be maternally transmitted in the family, and the onset of diabetes was occurring earlier and the insulin secretory capacity was declining from generation to generation, so that these findings suggest that the point mutation at np 3316 is associated with various phenotypes of diabetes.
\end{abstract}

Key words: Mitochondrial DNA, Point mutation, Nucleotide pair 3316, Non-insulin-dependent diabetes mellitus (NIDDM), IDDM

(Endocrine Journal 45: 625-630, 1998)

MANY kinds of mutations and deletions in mitochondrial DNA (mtDNA) have been reported to be associated with the development of diabetes mellitus in concert with other genetic factors and/ or environmental factors [1-4]. Among them,

Received: February 2, 1998

Accepted: May 27, 1998

Correspondence to: Dr. Shigeru NAKANO, Division of Endocrinology, Department of Internal Medicine, Kanazawa Medical University, 1-1 Daigaku, Uchinada, Ishikawa 920-0293, Japan
tRNA ${ }^{\text {Lue(UUR) }}$ and its adjacent region within the mitochondrial genome are linked to high susceptibility to diabetes mellitus [2]. A point mutation at nucleotide pair (np) 3243 in the mitochondrial tRNA ${ }^{\text {Lue(UUR) }}$ gene is commonly referred to as MELAS (mitochondrial myopathy, encephalopathy, lactic acidosis and stroke-like episodes), and has been recognized as a causative factor in a new clinical entity, i.e. maternally inherited diabetes and deafness (MIDD) $[5,6]$. Mitochondrial dysfunction through the involvement of mitochondrial oxidative phosphorylation (OXPHOS) may lead to 
impairment of glucose-induced insulin secretion from pancreatic $\beta$ cells in subjects harboring these mutations $[2,3,7]$. The clinical phenotypes of diabetes mellitus with these mutations are generally heterogeneous $[5,8]$. Recently, we found a mtDNA mutation at $\mathrm{np} 3316$ in all of family members with different phenotypes of diabetes. The proband and the proband's family are presented here with special reference to possible mechanisms causing different types of diabetes.

\section{Subjects and Methods}

\section{Subjects}

A 49-year-old woman, the proband in a family with a mtDNA point mutation at np 3316, was referred to our hospital on May 10, 1996 for treatment of the diabetic gangrene in her left foot. She had an 11-year history of non-insulindependent diabetes mellitus (NIDDM) treated with 28 units of intermediate-acting insulin per day since her family doctor first detected hyperglycemia. She had never experienced ketoacidosis, ketonuria, a stroke-like episode or hearing impairment before her admission. The pedigree is shown in Fig. 1. There was a family history of diabetes in her two sons, a pair of 20-year-old single-ovum-twins who had been receiving insulin therapy since they were both diagnosed with insulin-dependent diabetes mellitus (IDDM) because of hyperglycemic coma with ketoacidosis at the age of 7 . The proband's father was not diabetic but died of an uncertain cause at age 50, and her husband was died of nondiabetic renal failure at the age 42 . Her mother is living and well of 68 years old.

On physical examination, she was not obese (body mass index (BMI); $23.6 \mathrm{Kg} / \mathrm{m}^{2}$ ). Preproliferative diabetic retinopathy was seen in both optic fundi and reduced tendon reflexes were found in the lower legs. An auditory test was normal. The left foot was markedly swollen and intensely purulent with severe tenderness, suggesting the presence of diabetic gangrene. The remainder of the physical examination was unremarkable.

Laboratory data on admission were as follows. Urinalysis showed $3+$ for sugar, $1+$ for protein, and $3+$ for ketone body. The microscopic
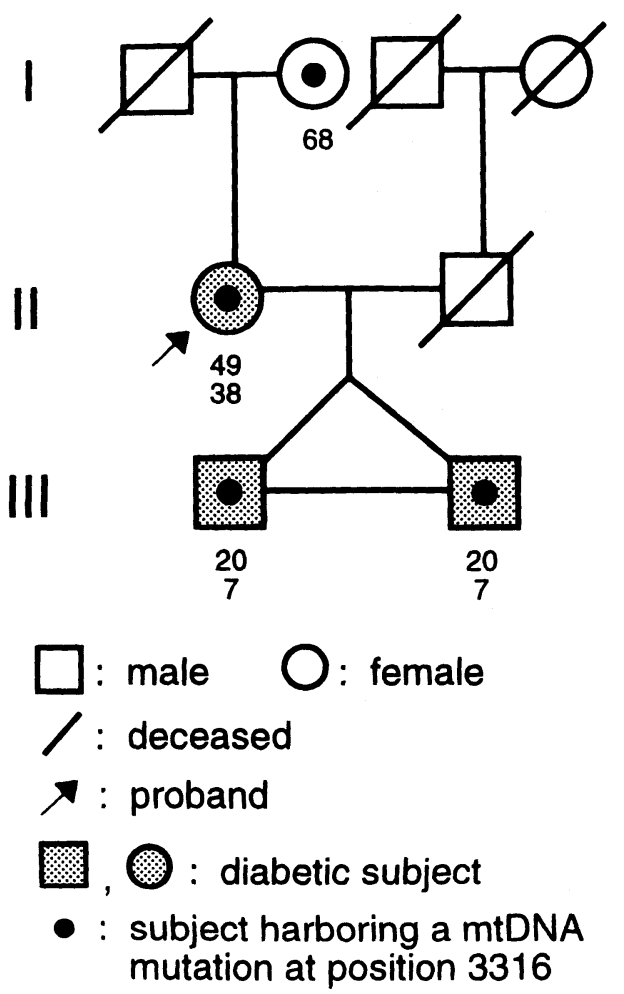

Fig. 1. The pedigree of the family. Roman numerals indicate generations. The proband is indicated by an arrow. The first line below the symbols are the ages of family members. The second line are the ages at the onset of diabetes mellitus.

examination of the urinary sediment revealed 10 to 20 white blood cells per high-power field. The white blood cell count was $19,870 / \mathrm{mm}^{3}$ with a left shift of granulocytes. The sedimentation rate was $91 \mathrm{~mm}$ in one hour, and the C-reactive protein was markedly positive. Fasting plasma glucose and $\mathrm{HbA1c}$ were $18.0 \mathrm{mmol} / \mathrm{L}$ and $13.8 \%$, respectively. Serum electrolytes and serum lipids were all within normal limits. Renal and liver functions were normal. Blood gas analysis was also normal. Islet cell antibodies (ICA), glutamic acid decarboxylase (GAD) antibody, and insulin antibodies were later found to be negative. The audiogram, electrocardiogram, and ultracardiosonogram showed nothing particular, and the brain computed tomographic scan also showed a normal image.

The administration of antibiotics combined with intensive insulin therapy was immediately started, the skin lesion of her left foot was debrided, and a complete recovery was obtained within two 
months.

After recovery from the gangrene, her insulin secretory capacity was evaluated. Urinary Cpeptide secretion was considered to be low (7.1 nmol/day, normal range; 19.9-39.7). Serum Cpeptide reactivity 6 min after an i.v. injection of 1 $\mathrm{mg}$ glucagon was also low $(0.3 \mathrm{nmol} / \mathrm{L})$. Nevertheless, her insulin sensitivity was normal with a constant glucose infusion rate (a $M$ value) of $40.6 \mu \mathrm{mol} \cdot \mathrm{Kg}$ of body weight ${ }^{-1} \cdot \mathrm{min}^{-1}$ (normal; $46.9 \pm 7.4$ [mean \pm SD] in our laboratory) evaluated by the euglycemic hyperinsulinemic glucose clamp technique of DeFronzo [9].

The clinical status of other family members was later examined. BMI values of the proband's mother and twin sons were 22.8, 20.5 and $21.3 \mathrm{Kg} /$ $\mathrm{m}^{2}$, respectively, Autoantibodies such as ICA, GAD antibody and insulin antibodies were also negative in them. Furthermore, the proband's mother showed a normal pattern in a $75 \mathrm{~g}$ oral glucose tolerance test accompanying a normal insulin response with a peak value of $258.3 \mathrm{pmol} / \mathrm{L}$ at 30 min, and serum C-peptide levels $6 \mathrm{~min}$ after an i.v. injection of $1 \mathrm{mg}$ glucagon in the twin sons were 0.2 and $0.1 \mathrm{nmol} / \mathrm{L}$.

\section{MtDNA analysis}

MtDNA was isolated from peripheral leukocytes of patients with a Genomic DNA extraction kit (Talent srl, Trieste, Italy), and mtDNA fragments were amplified by polymerase chain reaction (PCR) with sense primer 5'-CAA CCT CCG AGC AGT ACA TG-3' (from np 2836 to 2855) and antisense primer 5'-CAA TGA GGA GTA GGA GGT TGG-3' (from np 3317 to 3337). The PCR mixture contained $0.2 \mathrm{mM}$ dNTP, $1.0 \mathrm{mM}$ primers, 0.625 unit rTaq polymerase, $0.2 \mu \mathrm{g}$ sample DNA in a $25 \mu \mathrm{l}$ final volume. The PCR conditions were as follows; initial denaturation at $94^{\circ} \mathrm{C}$ for $5 \mathrm{~min}$, then 60 cycles of denaturation at $95^{\circ} \mathrm{C}$ for $30 \mathrm{sec}$, annealing at $60^{\circ} \mathrm{C}$ for $30 \mathrm{sec}$, and extension at $68^{\circ} \mathrm{C}$ for $1 \mathrm{~min}$ without a final extension under a different condition. PCR products were digested with restriction endonuclease Hae III at $37^{\circ} \mathrm{C}$ for $2 \mathrm{~h}$, and subjected to agarose gel ( 2.0 percent) electrophoresis in a TBE buffer containing $20 \mu \mathrm{g} / \mathrm{d} l$ of ethidium bromide. Separated DNA fragments were fluorometrically detected by ultraviolet irradiation. When the mutation was present, fragments of mtDNA were amplified by PCR with the same primers, and then directly sequenced with an autosequencer, ABI PRISM $^{\mathrm{TM}} 310$ GENETIC ANALYZER (Perkin-Elmer Co., Norwalk, Connecticut, USA).

\section{Responses of serum lactate, pyruvate and ketones (acetoacetate and $\beta$-hydroxybutyrate) to a $5 \mathrm{~min}$ ambulation}

Serum lactate, pyruvate and ketones (acetoacetate and $\beta$-hydroxybutyrate) before and after a $5 \mathrm{~min}$ ambulation were measured as a marker of mitochondrial function, and compared with those in age- and sex-matched nondiabetic (control) subjects $(n=5$; range $44-58$ years; mean $\pm S D, 49.4$ \pm 5.5 years). All control subjects were also informed of the research character of this study and gave their consent. Serum lactate, pyruvate and ketones were determined enzymatically.

\section{Results}

The patterns of agarose gel electrophoresis in mtDNA analysis with proband's peripheral leukocytes are shown in Fig. 2. A band for nondigested PCR products was located at 502 base pair (bp) in length (Fig. 2A, lane 1), and Hae III-digested wild-type mtDNAs were separated into three bands; at positions $312 \mathrm{bp}$ (through np 2836 to 3147), $169 \mathrm{bp}$ (through np 3148 to 3316) and $21 \mathrm{bp}$ (through np 3317 to 3337 , not visible) in length (Fig. 2A, lane 2), but bands for PCR products from the proband were located at 312 and $190 \mathrm{bp}$ in length without heteroplasmy for the mutation (Fig. 2A, lane 3). By the direct nucleotide sequence, a G-to-A transition at np 3316 of mtDNA, causing an amino acid replacement from alanine to threonine, was identified in this subject as a proband (Fig. 2B). The same homoplasmic mutations were observed in peripheral leukocytes of the proband's mother and twin sons. Screening for a point mutation at $\mathrm{np} 3243$ was negative in all family members.

In the proband, basal levels of serum lactate, pyruvate and ketones (acetoacetate and $\beta$ hydroxybutyrate) were within the normal range, but serum lactate alone markedly increased from 0.89 to $3.23 \mathrm{mmol} / \mathrm{L}$ after a $5 \mathrm{~min}$ ambulation over the levels demonstrated in control subjects (Fig. 3). 
A

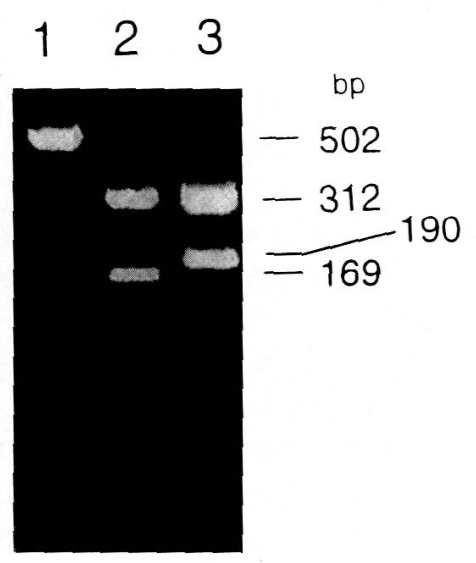

B
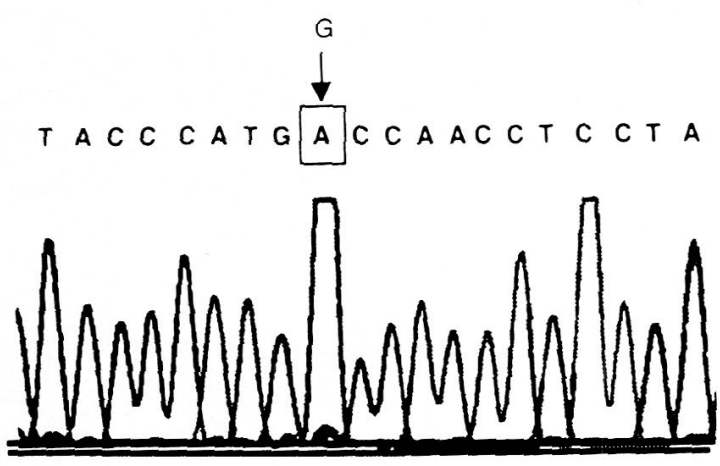

Fig. 2. A: Photograph of agarose gel containing ethidium bromide stained mtDNA fragments under ultraviolet light. Lane 1 shows a band for nondigested PCR products located at $502 \mathrm{bp}$ in length. Lane 2 shows Hae III-digested wild-type mtDNAs separated into three bands; at positions 312, 169 and $21 \mathrm{bp}$ (not visible) in length. Lane 3 shows bands for PCR products, obtained from the proband, located at 312 and $190 \mathrm{bp}$ in length without heteroplasmy for the mutation. B: The sequences of mtDNA surrounding the mutated site in the proband. By the direct nucleotide sequence, a G-to-A transition at np 3316 of mtDNA was identified in this subject as a proband.

\section{Discussion}

In this report, we describe a non-obese family with a G-to-A transition at np 3316 of mtDNA probably transmitted maternally over three generations in the pedigree. The mutation was shown to be homoplasmic in the proband, her twin sons and mother, and lost a recognition site for restriction endonuclease Hae III. The proband had an insulin-requiring NIDDM with low insulin secretory capacity but normal insulin sensitivity.

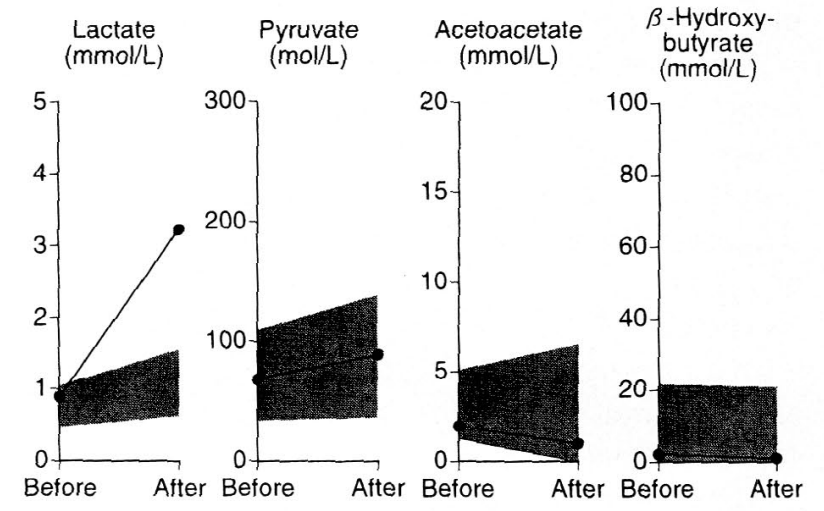

Fig. 3. Changes in serum levels of lactate, pyruvate and ketones (acetoacetate and $\beta$-hydroxybutyrate) before and after a $5 \mathrm{~min}$ ambulation of the proband (O) and control subjects (shaded area; mean \pm SEM).

The proband's twin sons had been diagnosed as having IDDM, but the proband's mother was not diabetic at this time. As none of the family members had any hearing loss or neuromuscular deficits other than the proband's peripheral polyneuropathy commonly observed as a diabetic microvascular complication, it seemed unlikely that they were affected by the related syndromes usually observed in patients with point mutations of mtDNA at np 3243 or its adjacent region.

Mitochondrial genes are beginning to be recognized as candidate genes for diabetes [2-6, 8]. Although new mutations have recently been detected in association with the development of diabetes mellitus, the mtDNA point mutation at np 3243 is clearly demonstrated to be correlated with diabetes mellitus $[5,8]$. The prevalence of mtDNA at np 3243 was reported to be less than $1 \%$ in the general population $[3,5]$, and higher prevalence was reported in subgroups of subjects with family histories of maternal diabetes, early onset of diabetes, and associated hearing loss and/ or neuromuscular defects $[5,8]$. On the other hand, the point mutation at $n p 3316$ of mtDNA is reported to be more frequent than that of the 3243 mutation. Nakagawa et al. [10] and McCarthy et al. [11] showed that the frequencies of point mutation at np 3316 were about $3 \%$ and $1 \%$ in randomly selected diabetic and control subjects, respectively. Moreover, most of the mutations analyzed with peripheral leukocytes in their studies were 
homoplasmic, just as they were in our observations. Although it is not known whether point mutations in pancreatic $\beta$ cells and neuromuscular cells are also homoplasmic in our own cases and previous cases, this is the first report demonstrating subjects harboring a $\mathrm{np} 3316$ point mutation as a family.

The clinical characteristics of diabetes mellitus in this family mimicked those of diabetic subjects harboring a point mutation at np $3243[5,8,12$, 13]. That is, they were non-obese, and the onset of diabetes was earlier and the insulin secretory capacity lower in successive generations of this family.

The np 3316 in the mtDNA belongs to the ND1encoding region which constitutes the OXPHOS system in the mitochondria [2]. The mtDNA mutation of this region is known to be linked with Leber's hereditary optic neuropathy (LHON), Altzheimer's disease and Parkinson's disease as well as diabetes [4,14]. Although the functional roles of mtDNA mutations seem to be unclear with regard to functional alterations in OXPHOS capacity to the point of disease manifestation, the close relationships between mtDNA mutations and the development of diabetes mellitus have been demonstrated [2]. In subjects with a mtDNA mutation, serum lactate levels were reported to be increased at rest and more pronounced during exercise, whereas abnormal serum pyruvate levels at rest and during exercise were shown to be less frequent that in corresponding lactate results [15, 16]. In this report, an abnormal increase in the serum lactate, but not the serum pyruvate and ketones, was exhibited in the proband after only a 5 min ambulation was probably due to functional alterations in ATP generation via the OXPHOS system in the mitochondria. Although a $5 \mathrm{~min}$ ambulation is not considered to be standardized, our findings therefore suggest that measurement of the serum lactate level before and after even a breif ambulation is useful in screening for mtDNA mutations.

In the family examined, the proband's mother is not diabetic, and phenotypes of diabetes are different in the proband and her twin sons. Although phenotypic variability between NIDDM and IDDM may be due to the degree of heteroplasmy for the point mutation in different tissues with different thresholds [17, 18], homoplasmy in peripheral leukocytes may not be strongly indicative of heteroplasmy in other organs such as pancreatic $\beta$ cells. It seems likely that other epigenic factors which are mitochondrial or nuclear in origin influence the penetrance and expression of syndromes such as diabetes mellitus. As another explanation for the occurrence of IDDM, autoimmune mechanisms may be provoked through this kind of mtDNA point mutation [13]. In fact, ICA-positive diabetic subjects harboring a mtDNA mutation at np 3243 were reported to show progress in insulin-dependency within a relatively short term, similar to that shown in slowly progressive IDDM subjects [19]. Gerbitz [13] pointed out that changes in mtDNA-encoded peptides may contribute to the diversity of antigens on the surface of pancreatic $\beta$ cells, leading to subsequent autoimmune responses. However, since no evidence based on autoimmunity was detected in these family members, coincidental occurrence of different types of diabetes in this family is also possible.

A rare pathogenic point mutation of mtDNA at np 3316 was identified in this family with heterogeneous types of diabetes, and disease segregation is probably consistent with maternal transmission. None of them had clinical evidence of neurological disease except for diabetic neuropathy, and the proband's mother was not diabetic at the time of her examination, but she might express susceptibility to diabetes in the future. Since the mtDNA mutation at np 3316 may play a role in the pathogenesis of diabetes mellitus, further follow-up observations are needed to establish the clinical significance of this rare mtDNA point mutation, including analyses of environmental and epigenic factors.

\section{Acknowledgment}

This work was in part supported by a Grant for Project Research from Kanazawa Medical University (P96-1). 


\section{References}

1. Horai S, Matsunaga E (1986) Mitochondrial DNA polymorphism in Japanese. II. Analysis with restriction enzymes of four or five base pair recognition. Hum Genet 72: 105-117.

2. Gerbitz K, Gempel K, Brdiczka D (1996) Mitochondria and diabetes. Genetic, biochemical, and clinical implications of the cellular energy circuit. Diabetes 45: 113-126.

3. Gerbitz KD, van den Ouweland JM, Maassen JA, Jaksch M (1995) Mitochondrial diabetes mellitus: A review. Biochim Biophys Acta 37: 253-260.

4. Suomalainen A (1997) Mitochondrial DNA and disease. Ann Med 17: 235-246.

5. Maassen JA, Kadowaki T (1996) Maternally inherited diabetes and deafness: A new diabetes subtype. Diabetologia 39: 375-382.

6. van den Ouweland J, Lemkes J, Gerbitz K, Maassen J (1995) Maternally inherited diabetes and deafness (MIDD): A distinct subtype of diabetes associated with a mitochondrial tRNALue(UUR) gene point mutation. Muscle Nerve (Suppl) 3: S124-S130.

7. Sener A, Malaisse W (1987) Stimulation by Dglucose of mitochondrial oxidative events in ilet cells. Biochem J 246: 89-95.

8. Kadowaki T, Kadowaki H, Mori Y, Tobe K, Sakuta R, Suzuki Y, Tanabe Y, Sakura H, Awata T, Goto Y, Hayakawa T, Mtsuoka K, Kawamori R, Kamada T, Horai S, Nonaka I, Hagura R, Akanuma Y, Yazaki $Y$ (1994) A subtype of diabetes mellitus associated with a mutation of mitochondrial DNA. $N$ Engl J Med 330: 962-968.

9. DeFronzo RA, Tobim JD, Andres R (1979) Glucose clamp technique: A method for quantifying insulin secretion and resistance. Am J Physiol 237: E214E223.

10. Nakagawa $Y$, Ikegami $H$, Yamato E, Takekawa $K$, Fujisawa T, Hamada $Y$, Ueda H, Uchigata $Y$, Miki $T$, Kumahara $Y$, Ogihara $T$ (1995) A new mitochondrial DNA mutation associated with noninsulin-dependent diabetes mellitus. Biochem Biophys Res Commun 209: 664-668.

11. McCarthy $M$, Cassell $P$, Tran $T$, Mathias $L$, ' $t$ Hart L, Maassen J, Snehalatha C, Ramachandran A,
Viswanathan M, Hitman G (1996) Evaluation of the importance of maternal history of diabetes and of mitochondrial variation in the development of NIDDM. Diabetic Med 13: 420-428.

12. Otabe S, Sakura H, Shimokawa K, Mori $Y$, Kadowaki H, Yasuda K, Nonaka K, Hagura R, Akanuma Y, Yazaki Y, Kadowaki T (1994) The high prevalence of the diabetic patients with a mutation in the mitochondrial gene in Japan. J Clin Endocrinol Metab 79: 768-771.

13. Katagiri $H$, Asano $T$, Ishihara $H$, Inukai $K$, Anai $M$, Yamanouchi T, Tsukuda K, Kikuchi M, Kitaoka H, Ohsawa N, Yazaki Y, Oka Y (1994) Mitochondrial diabetes mellitus: Prevalence and clinical characterization of diabetes due to mitochondrial tRNA(Leu(UUR)) gene mutation in Japanese patients. Diabetologia 37: 504-510.

14. Gerbitz K (1992) Does the mitochondrial DNA play a role in the pathogenesis of diabetes? Diabetologia 35: 1181-1186.

15. Trijbels J, Sengers R, Ruitenbeek W, Fischer J, Bakkeren J, Janssen A (1988) Disorders of the mitochondrial respiratory chain: Clinical manifestations and diagnostic approach. Eur $J$ Pediatr 148: 92-97.

16. Dengler R, Wohlfarth K, Zierz S, Jobges M, Schubert $M$ (1996) Muscle fatigue, lactate, and pyruvata in mitochondrial myopathy with progressive external ophthalmoplegia. Muscle Nerve 19: 456-462.

17. Gerbitz KD, Paprotta A, Jaksch M, Zierz S, Drechsel $J$ (1993) Diabetes mellitus is one of the heterogeneous phenotypic features of a mitochondrial DNA point mutation within the tRNALeu(UUR) gene. FEBS Lett 321: 194-196.

18. Reardon W, Ross RJ, Sweeney MG, Luxon LM, Pembrey ME, Harding AE, Trembath RC (1992) Diabetes mellitus associated with a pathogenic point mutation in mitochondrial DNA. Lancet 340: 13761379.

19. Oka Y, Katagiri H, Yazaki Y, Murase T, Kobayashi $T$ (1993) Mitochondrial gene mutation in islet-cellantibody-positive patients who were initially non-insulin-dependent diabetics. Lancet 37: 527-528. 